\author{
Silva C. ${ }^{*}$, Portugal F.*, Carvalhas J.*, Alves C.* \\ *Centro Hospitalar e Universitário de Coimbra
}

\title{
Background:
}

In Portugal c-section rate is $\mathbf{3 2 , 9 \%} .{ }^{1}$ Although regional anaesthesia (RA) is the hallmark choice for c-section, general anaesthesia (GA) may be indicated due to emergent/urgent delivery, contraindication for regional technique or inadequate regional block.

The WHO suggests an ideal rate of $5-10 \%$ of c-section deliveries for optimal mother and child outcomes. ${ }^{2}$

\section{Goal of study:}

Characterize women that underwent GA for c-section in a tertiary obstetric unit, from january 2015 to october 2017.

\section{Material and methods:}

Review of clinical data of consecutive women submitted to c-section under GA in the considered time frame.

Retrospective and descriptive analysis were performed.

\section{Results:}

In this period there were 7057 deliveries and the c-section rate was $\mathbf{2 6 , 9 \%}$

- $12.8 \%$ ( $n=242)$ underwent general anaesthesia

- median age was $32,5 \pm 5,9$ years

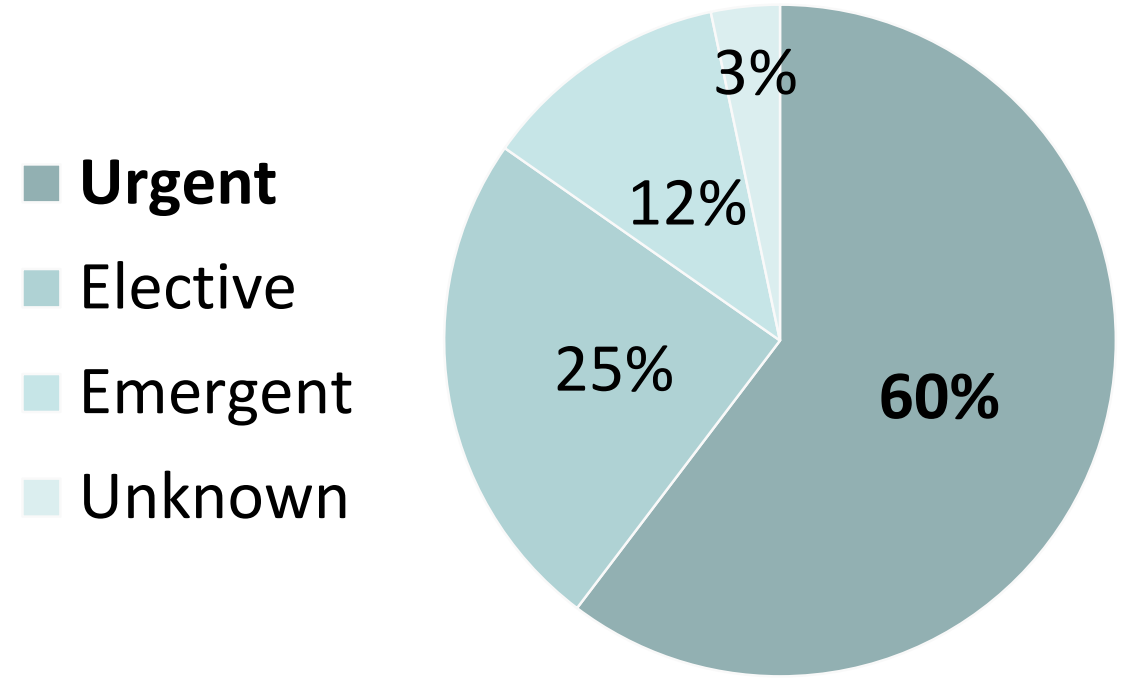

Graphic 1 - Classification of urgency $(n=242)$
- gestational age was $37 \pm 3,7$ weeks

- $75 \%$ were ASA II and $23 \%$ were ASA III

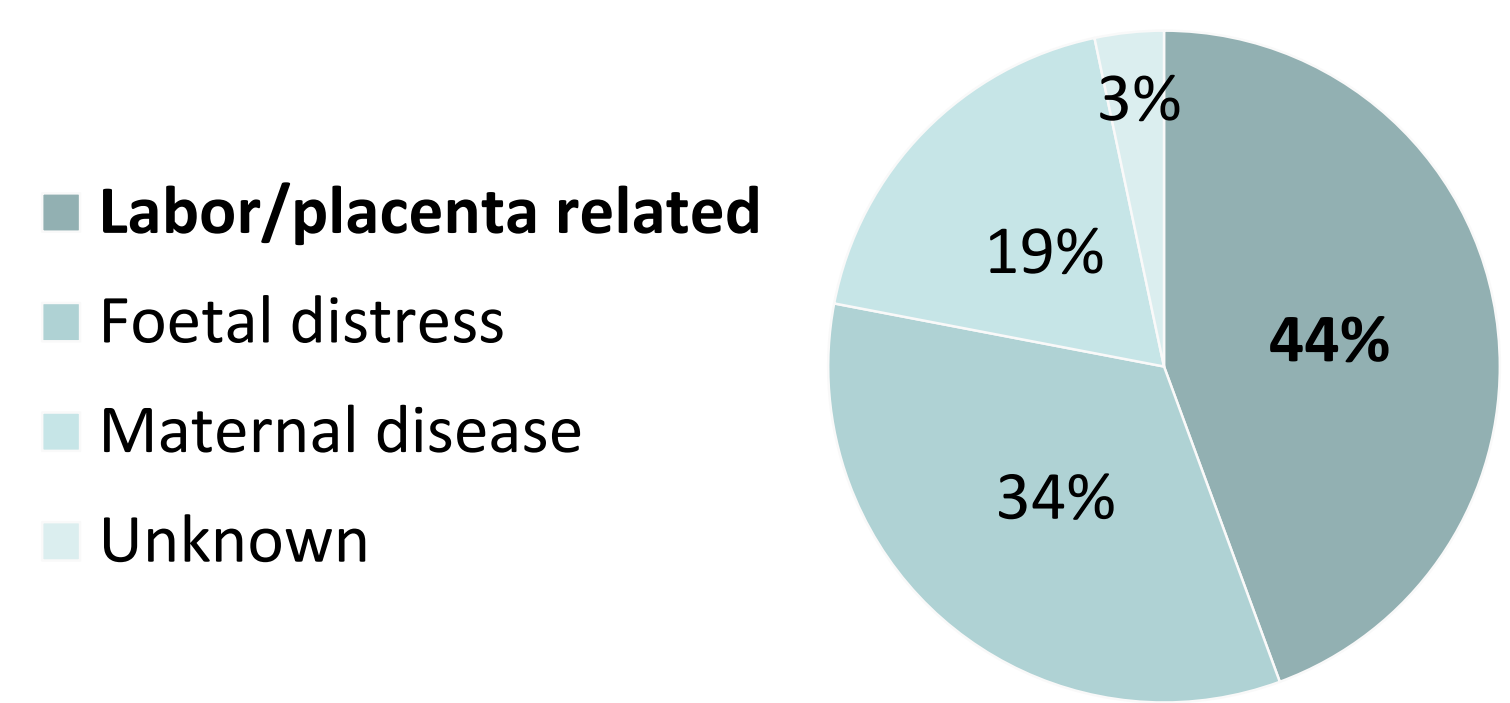

Graphic 2 - Indications for c-section $(n=242)$

Table 1 - Indications for general anaesthesia $(n=242)$

\begin{tabular}{ccccc}
\hline $\begin{array}{c}\text { Contraindication to regional } \\
\text { anaesthesia (38\%) }\end{array}$ & $\begin{array}{c}\text { Obstetric emergency } \\
\text { or urgency (29\%) }\end{array}$ & $\begin{array}{c}\text { Failure of regional } \\
\text { anaesthesia (17\%) }\end{array}$ & $\begin{array}{c}\text { Maternal refusal } \\
\text { for RA (7\%) }\end{array}$ & $\begin{array}{c}\text { Unknown } \\
\text { reason (9\%) }\end{array}$ \\
$\begin{array}{c}\text { Coagulation disorders 42\% } \\
\text { Active infection 16\% }\end{array}$ & $\begin{array}{c}\text { Foetal heart decelerations 40\% } \\
\text { Placental abruption 17\% }\end{array}$ & Epidural 55\% & - & - \\
$\begin{array}{c}\text { Lumbar spine pathology 11\% } \\
\text { Other chronic diseases 11\% }\end{array}$ & $\begin{array}{c}\text { Pre-eclampsia/HEELP 15\% } \\
\text { Prolapsed cord 6\% }\end{array}$ & Spinal 45\% & & - \\
\hline
\end{tabular}

$100 \%$ general balanced anaesthesia with rapid sequence induction:

- thiopental $75 \%$ and propofol $25 \%$

- suxamethonium $87 \%$ and rocuronium $13 \%$

- sevoflurane $100 \%$

\section{Conclusions and discussion:}

We observe a high rate of c-section and general anaesthesia. As a referral centre, we have a higher prevalence of maternal disorders and high-risk pregnancies contributing to contra-indicate regional anaesthesia. As a teaching unit for anaesthesia trainees, the prevalence of failed technique is probably inflated. 